\title{
On the error in the two-term Weyl formula for the Dirichlet Laplacian
}

Cite as: J. Math. Phys. 61, 043504 (2020); https://doi.org/10.1063/1.5145003

Submitted: 13 January 2020 . Accepted: 24 March 2020 . Published Online: 20 April 2020

Rupert L. Frank (D), and Simon Larson (D)

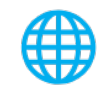




\title{
On the error in the two-term Weyl formula for the Dirichlet Laplacian
}

Cite as: J. Math. Phys. 61, 043504 (2020); doi: 10.1063/1.5145003

Submitted: 13 January 2020 - Accepted: 24 March 2020 •

Published Online: 20 April 2020

\author{
Rupert L. Frank ${ }^{1,2, a)}$ (D) and Simon Larson ${ }^{2, b)}$ (D)

\section{AFFILIATIONS} \\ ${ }^{1}$ Mathematisches Institut, Ludwig-Maximilans Universität München, Munich, Germany \\ ${ }^{2}$ Department of Mathematics, California Institute of Technology, Pasadena, California 91125, USA
}

Note: This paper is part of the Special Issue on Celebrating the Work of Jean Bourgain.

a) Electronic addresses: r.frank@lmu.de and rlfrank@caltech.edu

b) Author to whom correspondence should be addressed: larson@caltech.edu

\section{ABSTRACT}

We study the optimality of the remainder term in the two-term Weyl law for the Dirichlet Laplacian within the class of Lipschitz regular subsets of $\mathbb{R}^{d}$. In particular, for the short-time asymptotics of the trace of the heat kernel, we prove that the error term cannot be made quantitatively better than little- $o$ of the second term.

\section{Published under license by AIP Publishing. https://doi.org/10.1063/1.5145003}

\section{INTRODUCTION AND MAIN RESULTS}

Let $-\Delta_{\Omega}$ denote the Dirichlet Laplace operator on an open set $\Omega \subset \mathbb{R}^{d}$, which is defined as a self-adjoint operator in $L^{2}(\Omega)$ through the quadratic form $u \mapsto \int_{\Omega}|\nabla u(x)|^{2} d x$ with form domain $H_{0}^{1}(\Omega)$. If the measure of $\Omega \subset \mathbb{R}^{d}$ is finite, the spectrum of $-\Delta_{\Omega}$ is discrete and consists of an infinite number of positive eigenvalues accumulating only at infinity. Here, the eigenvalues are denoted by

$$
0<\lambda_{1} \leq \lambda_{2} \leq \lambda_{3} \leq \cdots,
$$

where each eigenvalue is repeated according to its multiplicity.

The study of the asymptotic behavior of $\lambda_{k}$ as $k \rightarrow \infty$ is a classical topic in spectral theory. The most fundamental result in this area is the following celebrated result going back to Weyl, ${ }^{1}$ which states that

$$
\#\left\{\lambda_{k}<\lambda\right\}=\frac{\omega_{d}}{(2 \pi)^{d}}|\Omega| \lambda^{d / 2}+o\left(\lambda^{d / 2}\right) \quad \text { as } \lambda \rightarrow \infty .
$$

Here and in what follows, $\omega_{d}$ denotes the volume of the $d$-dimensional unit ball. That (1) holds for any open set $\Omega \subset \mathbb{R}^{d}$ of finite measure was obtained in Ref. 2.

If the set $\Omega$ has certain geometric properties, a refined version of the asymptotic expansion (1) holds, namely,

$$
\#\left\{\lambda_{k}<\lambda\right\}=\frac{\omega_{d}}{(2 \pi)^{d}}|\Omega| \lambda^{d / 2}-\frac{1}{4} \frac{\omega_{d-1}}{(2 \pi)^{d-1}} \mathcal{H}^{d-1}(\partial \Omega) \lambda^{(d-1) / 2}+o\left(\lambda^{(d-1) / 2}\right) \quad \text { as } \lambda \rightarrow \infty .
$$

Here and in what follows, $\mathcal{H}^{d-1}(A)$ denotes the $(d-1)$-dimensional Hausdorff measure of a set $A \subset \mathbb{R}^{d}$. This refinement of Weyl's law was conjectured already by Weyl. ${ }^{3}$ A satisfactory answer remained elusive for several decades, but Ivrii ${ }^{4}$ proved the conjecture under the assumption that $\Omega$ is smooth and the measure of the periodic billiards in $\Omega$ is zero. 


\section{A. Main results}

In this paper, our focus is on the remainder term in (2) or rather the corresponding remainder term in certain averages of the counting function. The greater part of our analysis concerns the remainder term in the Abel-type average

$$
\operatorname{Tr}\left(e^{t \Delta_{\Omega}}\right)=\sum_{k \geq 1} e^{-t \lambda_{k}}=(4 \pi t)^{-d / 2}\left(|\Omega|-\frac{\sqrt{\pi t}}{2} \mathcal{H}^{d-1}(\partial \Omega)+o(\sqrt{t})\right) \quad \text { as } t \rightarrow 0^{+},
$$

that is, the short-time asymptotics of the trace of the heat kernel. The asymptotics (3) can be obtained from (2) by integration in $\lambda$. However, it is not possible to reverse this process and deduce (2) from (3) alone.

Intuitively, the averaging of the eigenvalues should have a regularizing effect on the asymptotics, and thus, one expects (3) to be valid under less restrictive geometric assumptions than those needed for (2). Under the weak assumption that the boundary of $\Omega$ is Lipschitz regular, the validity of (3) was proved by Brown. ${ }^{5}$ In the same paper, Brown remarked without proof that the error term $o(\sqrt{t})$ cannot be replaced by $o\left(t^{1 / 2+\epsilon}\right)$ for any $\epsilon>0$. The main theorem of this paper goes in the same direction as this remark and, in fact, contains the remark as a particular case. However, our result claims substantially more. While Brown's remark concerns the impossibility of improving the error term on the algebraic scale, our result states that it is impossible to make any quantitative improvement whatsoever. Specifically, we prove the following theorem:

Theorem 1.1. Let $g: \mathbb{R}_{+} \rightarrow \mathbb{R}$ be a non-negative function with $\lim _{t \rightarrow 0^{+}} g(t)=0$. There exists an open, bounded, and connected set $\Omega \subset \mathbb{R}^{d}$ with Lipschitz regular boundary such that

$$
\lim _{t \rightarrow 0^{+}} \frac{(4 \pi t)^{d / 2} \operatorname{Tr}\left(e^{t \Delta_{\Omega}}\right)-|\Omega|+\frac{\sqrt{\pi t}}{2} \mathcal{H}^{d-1}(\partial \Omega)}{\sqrt{\operatorname{tg}}(t)}=\infty .
$$

In addition to considering the trace of the heat kernel (3), we will consider the so-called Riesz means of order $\gamma \geq 0$, which are defined by

$$
\operatorname{Tr}\left(-\Delta_{\Omega}-\lambda\right)_{-}^{\gamma}=\sum_{\lambda_{k}<\lambda}\left(\lambda-\lambda_{k}\right)^{\gamma}, \quad \text { for } \lambda \geq 0
$$

where $x_{ \pm}=\frac{1}{2}(|x| \pm x)$. In particular, $\operatorname{Tr}\left(-\Delta_{\Omega}-\lambda\right)_{-}^{0}=\#\left\{\lambda_{k}<\lambda\right\}$. The quantities $\operatorname{Tr}\left(-\Delta_{\Omega}-\lambda\right)_{-}^{\gamma}$ become more and more well-behaved as $\gamma$ increases. Furthermore, by setting $\gamma=t \lambda$ and normalizing appropriately, one obtains $\operatorname{Tr}\left(e^{t \Delta_{\Omega}}\right)$ in the limit $\lambda \rightarrow \infty$.

Again by integration in $\lambda$, one can deduce a two-term asymptotic formula for the Riesz means from (2), which reads

$$
\operatorname{Tr}\left(-\Delta_{\Omega}-\lambda\right)_{-}^{\gamma}=L_{\gamma, d}|\Omega| \lambda^{\gamma+d / 2}-\frac{L_{\gamma, d-1}}{4} \mathcal{H}^{d-1}(\partial \Omega) \lambda^{\gamma+(d-1) / 2}+o\left(\lambda^{\gamma+(d-1) / 2}\right)
$$

as $\lambda \rightarrow \infty$, where we abbreviate

$$
L_{\gamma, d}=\frac{\Gamma(\gamma+1)}{(4 \pi)^{d / 2} \Gamma(\gamma+1+d / 2)} .
$$

As in the case of the trace of the heat kernel, the regularizing effect of averaging should help us to prove the validity of (5) in greater generality for larger $\gamma$. In a recent paper, ${ }^{6}$ it is proved that (5) is valid for $\gamma \geq 1$ as soon as the boundary of $\Omega$ is Lipschitz regular. This implies Brown's result, ${ }^{5}$ but not the other way around.

Our interest in the current topic was motivated by the question of sharpness for the result obtained in Ref. 6. As a corollary of Theorem 1.1, we obtain that the remainder $o\left(\lambda^{\gamma+(d-1) / 2}\right)$ in the asymptotic expansion in (5) cannot be improved.

Theorem 1.2. Let $R: \mathbb{R}_{+} \rightarrow \mathbb{R}$ be a non-negative function with $\lim _{\lambda \rightarrow \infty} R(\lambda)=0$. There exists an open, bounded, and connected set $\Omega \subset \mathbb{R}^{d}$ with Lipschitz regular boundary such that for all $\gamma \geq 0$,

$$
\limsup _{\lambda \rightarrow \infty} \frac{\operatorname{Tr}\left(-\Delta_{\Omega}-\lambda\right)_{-}^{\gamma}-L_{\gamma, d}|\Omega| \lambda^{\gamma+d / 2}+\frac{L_{\gamma, d-1}}{4} \mathcal{H}^{d-1}(\partial \Omega) \lambda^{\gamma+(d-1) / 2}}{\lambda^{\gamma+(d-1) / 2} R(\lambda)}=\infty .
$$

As Theorem 1.2 concerns the limsup and not the limit, the result is somewhat weaker than what could be expected from Theorem 1.1. As such, it is reasonable that it should follow from the same principal ideas. However, we are unable to give a direct proof. The main advantage in working with the trace of the heat kernel in comparison to $\operatorname{Tr}\left(-\Delta_{\Omega}-\lambda\right)_{-}^{\gamma}$ lies in that we can utilize pointwise estimates for the heat kernel. The corresponding estimates for $\operatorname{Tr}\left(-\Delta_{\Omega}-\lambda\right)_{-}^{\gamma}$ are much more delicate. However, it is not very surprising that one can deduce Theorem 1.2 from Theorem 1.1. Indeed, by the identity

$$
\operatorname{Tr}\left(e^{t \Delta_{\Omega}}\right)=\frac{t^{1+\gamma}}{\Gamma(1+\gamma)} \int_{0}^{\infty} \operatorname{Tr}\left(-\Delta_{\Omega}-\lambda\right)_{-}^{\gamma} e^{-t \lambda} d \lambda
$$


an asymptotic expansion for $\operatorname{Tr}\left(-\Delta_{\Omega}-\lambda\right)_{-}^{\gamma}$ as $\lambda \rightarrow \infty$ implies a corresponding expansion for $\operatorname{Tr}\left(e^{t \Delta_{\Omega}}\right)$ as $t \rightarrow 0^{+}$. However, the use of (6) in our Proof of Theorem 1.2 leads to the limsup instead of the limit.

\section{B. Additional remarks}

In a direction similar to that of Theorems 1.2 and 1.1, it was shown by Lazutkin and Terman ${ }^{7}$ that the error term in (2) cannot be improved on the algebraic scale even among planar convex sets, which, in addition, satisfy the assumptions of Ivrii's result. It is interesting to note that if one considers not the asymptotics of the counting function but of either $\operatorname{Tr}\left(-\Delta_{\Omega}-\lambda\right)_{-}^{\gamma}$, with $\gamma \geq 1$, or $\operatorname{Tr}\left(e^{t \Delta_{\Omega}}\right)$, one can improve the error term on the algebraic scale within convex sets (in any dimension). This is the case that can be deduced from the following uniform inequality proved by the authors in Ref. 6. There exists a constant $C>0$ such that for any convex domain $\Omega \subset \mathbb{R}^{d}$ and all $\lambda \geq 0$,

$$
\begin{aligned}
\left|\operatorname{Tr}\left(-\Delta_{\Omega}-\lambda\right)_{-}-L_{d}\right| \Omega \mid \lambda^{1+d / 2}+\frac{L_{d-1}}{4} & \mathcal{H}^{d-1}(\partial \Omega) \lambda^{1+(d-1) / 2} \mid \\
& \leq C \mathcal{H}^{d-1}(\partial \Omega) \lambda^{1+(d-1) / 2}(r(\Omega) \sqrt{\lambda})^{-1 / 11},
\end{aligned}
$$

where $r(\Omega)$ denotes the inradius of $\Omega$. The corresponding inequality for $\gamma>1$ and $\operatorname{Tr}\left(e^{t \Delta_{\Omega}}\right)$ follows from (7) through integration in $\lambda$.

For $\Omega \subset \mathbb{R}^{2}$, a piecewise smooth domain with finitely many corners, it is possible to refine the asymptotic expansions discussed by yet another term ${ }^{8-12}$ (see also Ref. 13 for a similar result in polyhedra in $\mathbb{R}^{d}$ ). Let the boundary of $\Omega$ be given by the union of smooth curve segments $\gamma_{j}, j=1, \ldots, m$, parameterized by the arc length and ordered so that $\gamma_{j}$ meets $\gamma_{j+1}$ and $\gamma_{m}$ meets $\gamma_{1}$. Let $\alpha_{j} \in(0,2 \pi)$ denote the interior angle formed at the point $\gamma_{j} \cap \gamma_{j+1}$. Then, as $t \rightarrow 0^{+}$,

$$
\operatorname{Tr}\left(e^{t \Delta_{\Omega}}\right)=(4 \pi t)^{-1}\left(|\Omega|-\frac{\sqrt{\pi t}}{2} \mathcal{H}^{1}(\partial \Omega)+\frac{t}{3} \sum_{j=1}^{m}\left[\int_{\gamma_{j}} \kappa(s) d s+\frac{\pi^{2}-\alpha_{j}^{2}}{2 \alpha_{j}}\right]+o(t)\right),
$$

where $\kappa(s)$ denotes the curvature. It is clear that this expansion cannot extend to the class of Lipschitz sets, as the third term need not be finite.

There has been interesting work on Weyl asymptotics in very irregular sets, specifically sets with fractal boundary. In particular, a lot of work has been directed toward the optimal order of the error term in (1) in this setting. In 1979, it was conjectured by Berry ${ }^{14,15}$ that if $\partial \Omega$ has the Hausdorff dimension $d-1<d_{\mathcal{H}} \leq d$, then

$$
\#\left\{\lambda_{k}<\lambda\right\}=\frac{\omega_{d}}{(2 \pi)^{d}}|\Omega| \lambda^{d / 2}+O\left(\lambda^{d_{\mathcal{H}} / 2}\right) \quad \text { as } \lambda \rightarrow \infty .
$$

However, it was shown by Brossard and Carmona ${ }^{16}$ that as stated, the conjecture of Berry needs to be modified, and they suggested that the Hausdorff dimension of $\partial \Omega$ should be replaced by the Minkowski dimension. Subsequently, it was proved by Lapidus ${ }^{17}$ that if $\partial \Omega$ has the Minkowski dimension $d_{\mathcal{M}}$ and a finite $d_{\mathcal{M}}$-dimensional Minkowski content, then the error in the Weyl formula is $O\left(\lambda^{d_{\mathcal{M}} / 2}\right)$. We emphasize, however, that the sets considered here, while having a non-trivial structure on all scales such as fractals, are much more regular. Nevertheless, the crucial feature of the set that we construct in the proof of Theorem 1.1 and 1.2 is that its boundary has non-trivial structure on all microscopic scales.

\section{PROOF OF THEOREM 1.1}

This section is dedicated to an outline of the Proof of Theorem 1.1. We defer the proof of two ingredients to Secs. III and IV. Our proof is based on the explicit construction of a Lipschitz domain $\Omega$, satisfying (4) for a given function $g$.

\section{A. Reduction to the two-dimensional case}

In order to simplify the construction, we first show that it is sufficient to consider the two-dimensional case. Assume that Theorem 1.1 is known in the case $d=2$. Let $g$ be as in the statement of Theorem 1.1 and fix a Lipschitz regular open, bounded, and connected set $\Omega \subset \mathbb{R}^{2}$, satisfying (4) with $g$ replaced by $\tilde{g}(t)=\max \left\{g(t), t^{1 / 2}\right\}$. We claim that $\Omega^{\prime}=\Omega \times(0,1)^{d-2}$ satisfies (4). Clearly, $\Omega^{\prime}$ is open, bounded, connected, and Lipschitz regular.

By the product structure of $\Omega^{\prime}$,

$$
\begin{aligned}
\operatorname{Tr}\left(e^{t \Delta_{\Omega^{\prime}}}\right) & =\sum_{l \geq 1} e^{-t \lambda_{l}\left(\Omega^{\prime}\right)}=\sum_{j, k \geq 1} e^{-t\left(\lambda_{k}(\Omega)+\lambda_{j}\left((0,1)^{d-2}\right)\right)} \\
& =\sum_{j, k \geq 1} e^{-t \lambda_{k}(\Omega)} e^{-t \lambda_{j}\left((0,1)^{d-2}\right)}=\operatorname{Tr}\left(e^{t \Delta_{\Omega}}\right) \operatorname{Tr}\left(e^{\left.t \Delta_{(0,1)}\right)^{d-2}}\right) .
\end{aligned}
$$

Moreover,

$$
\left|\Omega^{\prime}\right|=|\Omega| \quad \text { and } \quad \mathcal{H}^{d-1}\left(\partial \Omega^{\prime}\right)=\mathcal{H}^{1}(\partial \Omega)+2(d-2)|\Omega| \text {. }
$$


It is not difficult to show that

$$
\operatorname{Tr}\left(e^{t \Delta_{(0,1)^{d-2}}}\right)=(4 \pi t)^{-(d-2) / 2}\left(1-\frac{\sqrt{\pi t}}{2} 2(d-2)+O(t)\right) .
$$

In fact, an explicit computation based on the Poisson summation formula and the explicit formulas for the eigenfunctions and eigenvalues on the interval yields the following lemma:

Lemma 2.1. The Dirichlet heat kernel of $-\Delta_{(0, L)}$ evaluated on the diagonal is given by

$$
e^{t \Delta_{(0, L)}}(x, x)=\frac{1}{\sqrt{4 \pi t}} \sum_{m \in \mathbb{Z}}\left(e^{-\frac{m^{2} L^{2}}{t}}-e^{-\frac{(m L+x)^{2}}{t}}\right) .
$$

Proof of Lemma 2.1. By scaling, it suffices to consider the case $L=1$. Recall that $\lambda_{k}=\pi^{2} k^{2}$ with the corresponding $L^{2}$-normalized eigenfunction $\varphi_{k}(x)=\sqrt{2} \sin (\pi k x)$. By the explicit formulas for the eigenfunctions and eigenvalues,

$$
e^{t \Delta_{I}}(x, x)=2 \sum_{k=1}^{\infty} e^{-t \pi^{2} k^{2}} \sin ^{2}(\pi k x)=\sum_{k \in \mathbb{Z}} e^{-t \pi^{2} k^{2}} \sin ^{2}(\pi k x) .
$$

Applying the Poisson summation formula

$$
\sum_{k \in \mathbb{Z}} g(k)=\sqrt{2 \pi} \sum_{m \in \mathbb{Z}} \check{g}(2 \pi m)
$$

with $g(\xi)=e^{-t \pi^{2} \xi^{2}} \sin ^{2}(\pi \xi x)$ and using the fact that

$$
\check{g}(y)=\frac{1}{\sqrt{2 \pi}} \int_{\mathbb{R}} e^{-t \pi^{2} \xi^{2}} \sin ^{2}(\pi \xi x) e^{i y \xi} d \xi=\frac{1}{4 \pi \sqrt{2 t}}\left(2 e^{-\frac{y^{2}}{4 \pi^{2} t}}-e^{-\frac{(y-2 \pi x)^{2}}{4 \pi^{2} t}}-e^{-\frac{(y+2 \pi x)^{2}}{4 \pi^{2} t}}\right)
$$

complete the proof of the lemma.

By integrating (11), one obtains

$$
\operatorname{Tr}\left(e^{t \Delta_{(0,1)}}\right)=\int_{0}^{1} e^{t \Delta_{(0,1)}}(x, x) d x=(4 \pi t)^{-1 / 2}\left(1-\frac{\sqrt{\pi t}}{2} 2+O\left(e^{-1 / t}\right)\right) \quad \text { as } t \rightarrow 0^{+} .
$$

Thus, by the product structure of $(0,1)^{n}$,

$$
\begin{aligned}
\operatorname{Tr}\left(e^{t \Delta_{(0,1)^{n}}}\right)=\left(\operatorname{Tr}\left(e^{t \Delta_{(0,1)}}\right)\right)^{n} & =\left[(4 \pi t)^{-1 / 2}\left(1-\frac{\sqrt{\pi t}}{2} 2+O\left(e^{-1 / t}\right)\right)\right]^{n} \\
& =(4 \pi t)^{-n / 2}\left(1-\frac{\sqrt{\pi t}}{2} 2 n+O(t)\right)
\end{aligned}
$$

which is the claimed expansion (10).

Combined with $(4 \pi t) \operatorname{Tr}\left(e^{t \Delta_{\Omega}}\right)=|\Omega|+O(\sqrt{t})$ by Brown ${ }^{5}$ and (9), we find

$$
\begin{aligned}
(4 \pi t)^{d / 2} \operatorname{Tr}\left(e^{t \Delta_{\Omega^{\prime}}}\right) & -\left|\Omega^{\prime}\right|+\frac{\sqrt{\pi t}}{2} \mathcal{H}^{d-1}\left(\partial \Omega^{\prime}\right) \\
& =(4 \pi t) \operatorname{Tr}\left(e^{t \Delta_{\Omega}}\right)-|\Omega|+\frac{\sqrt{\pi t}}{2} \mathcal{H}^{1}(\partial \Omega)+O(t),
\end{aligned}
$$

so

$$
\begin{aligned}
& \lim _{t \rightarrow 0^{+}} \frac{(4 \pi t)^{d / 2} \operatorname{Tr}\left(e^{t \Delta_{\Omega^{\prime}}}\right)-\left|\Omega^{\prime}\right|+\frac{\sqrt{\pi t}}{2} \mathcal{H}^{d-1}\left(\partial \Omega^{\prime}\right)}{\sqrt{t} g(t)} \\
& \quad=\lim _{t \rightarrow 0^{+}} \frac{(4 \pi t) \operatorname{Tr}\left(e^{t \Delta_{\Omega}}\right)-|\Omega|+\frac{\sqrt{\pi t}}{2} \mathcal{H}^{1}(\partial \Omega)+O(t)}{\sqrt{t} \tilde{g}(t)} \frac{\tilde{g}(t)}{g(t)}=\infty,
\end{aligned}
$$

where in the last step, we used the choice of $\Omega$ and the fact that by construction, $\tilde{g}(t) \geq g(t)$ and $t^{1 / 2} \leq \tilde{g}(t)$. This proves our claim and consequently reduces the Proof of Theorem 1.1 to the two-dimensional case. 


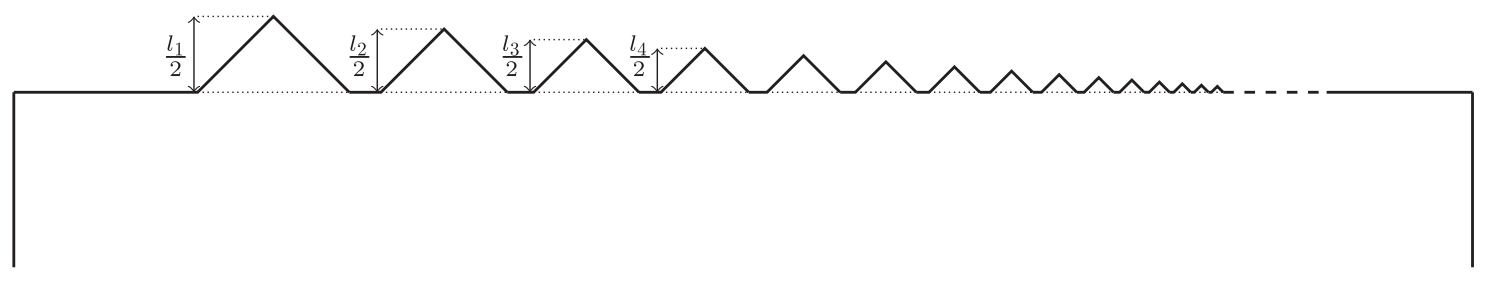

FIG. 1. A schematic illustration of the top part of the constructed set $\Omega$.

\section{B. Geometric construction}

We construct a domain $\Omega \subset \mathbb{R}^{2}$ as follows: The idea is to begin with the square $Q_{0}=(0,3)^{2}$ to which we add triangular teeth to the top edge, all separated from each other and of the same shape but of different sizes and all away from the vertical edges of the large square (see Fig. 1).

Precisely, we consider

$$
\Omega=\left\{\left(x_{1}, x_{2}\right) \in \mathbb{R}^{2}: 0<x_{1}<3,0<x_{2}<H\left(x_{1}\right)\right\}
$$

where

$$
H(x)=3+\sum_{k \geq 1} l_{k} H_{0}\left(\left(x-c_{k}\right) / l_{k}\right) \quad \text { with } H_{0}(x)= \begin{cases}x, & x \in[0,1 / 2] \\ 1-x, & x \in(1 / 2,1] \\ 0, & \text { otherwise, }\end{cases}
$$

where the sequence $\left\{l_{k}\right\}_{k \geq 1}$ is positive and non-increasing with $\sum_{k} l_{k} \leq 1$, and the $c_{k}$ are chosen increasing and such that $1 \leq c_{k} \leq$ min $\left\{c_{k+1}-l_{k}, 2\right\}$. This ensures that the supports of the different copies of $H_{0}$ are disjoint and at least a distance 1 away from the vertical edges of the square $Q_{0}$. For instance, the sequence given by $c_{1}=1$ and $c_{k}=1+\sum_{j=1}^{k-1} l_{j}$ for $k>1$ satisfies all the requirements. Note that at all points $x$, where $H$ is differentiable, we have $H^{\prime}(x) \in\{0,1,-1\}$. Thus, the set $\Omega$ defined by (12) is open, bounded, and connected and has Lipschitz regular boundary.

The idea is that the teeth at a scale much smaller than $\sqrt{t}$ should not have an essential contribution to the trace of the heat kernel. Moreover, the decrease in the area in removing such teeth is small relative to the decrease in the length of the boundary. We emphasize that it is not the presence of corners that we are playing with to construct our counterexample. The effect that is essential for our construction is rather that the boundary has a non-trivial structure on all scales. Heuristically, the construction should go through if the triangular teeth were replaced by versions, where each of the three corners had been smoothed out. However, even though such a modification would make $H_{0}$ smooth, the function $H$ and the boundary of the set $\Omega$ would be remain merely Lipschitz due to all derivatives of order greater than 1 blowing up as $l_{k}$ tends to zero.

Let $\Omega_{M}$ be the domain described similarly to $\Omega$ by (12), but with the function $H$ replaced by $H_{M}$,

$$
H_{M}(x)=3+\sum_{k<M} l_{k} H_{0}\left(\left(x-c_{k}\right) / l_{k}\right)
$$

That is, we remove all teeth after the $M$ th one. We note that $\Omega_{M^{\prime}} \subset \Omega_{M} \subset \Omega$, if $M^{\prime}<M, \cup_{M \geq 1} \Omega_{M}=\Omega$, and by construction,

$$
|\Omega|-\left|\Omega_{M}\right|=\sum_{k \geq M} \frac{l_{k}^{2}}{4}, \quad \mathcal{H}^{1}(\partial \Omega)-\mathcal{H}^{1}\left(\partial \Omega_{M}\right)=\sum_{k \geq M}(\sqrt{2}-1) l_{k} .
$$

By monotonicity of Dirichlet eigenvalues under set inclusions,

$$
\begin{aligned}
(4 \pi t) \operatorname{Tr}\left(e^{t \Delta_{\Omega}}\right)- & |\Omega|+\frac{\sqrt{\pi t}}{2} \mathcal{H}^{1}(\partial \Omega) \\
& \geq(4 \pi t) \operatorname{Tr}\left(e^{t \Delta_{\Omega_{M}}}\right)-|\Omega|+\frac{\sqrt{\pi t}}{2} \mathcal{H}^{1}(\partial \Omega) \\
& =\left|\Omega_{M}\right|-|\Omega|+\frac{\sqrt{\pi t}}{2}\left(\mathcal{H}^{1}(\partial \Omega)-\mathcal{H}^{1}\left(\partial \Omega_{M}\right)\right)+E_{M}(t),
\end{aligned}
$$

where we define

$$
E_{M}(t)=4 \pi t \operatorname{Tr}\left(e^{t \Omega_{M}}\right)-\left|\Omega_{M}\right|+\frac{\sqrt{\pi t}}{2} \mathcal{H}^{1}\left(\partial \Omega_{M}\right)
$$


Our goal is to choose $\left\{l_{k}\right\}_{k \geq 1}$ and $M$ and to bound $E_{M}(t)$ in such a way that

$$
\lim _{t \rightarrow 0^{+}}(\sqrt{t} g(t))^{-1}\left(\left|\Omega_{M}\right|-|\Omega|+\frac{\sqrt{\pi t}}{2}\left(\mathcal{H}^{1}(\partial \Omega)-\mathcal{H}^{1}\left(\partial \Omega_{M}\right)\right)+E_{M}(t)\right)=\infty
$$

Equivalently, by (14), we want to achieve

$$
\lim _{t \rightarrow 0^{+}} g(t)^{-1}\left(\frac{\sqrt{\pi}(\sqrt{2}-1)}{2} \sum_{k \geq M} l_{k}-\frac{1}{4 \sqrt{t}} \sum_{k \geq M} l_{k}^{2}+\frac{E_{M}(t)}{\sqrt{t}}\right)=\infty
$$

Before we are able to conclude our proof, we shall need to prove some auxiliary results. The first result that we shall need is a bound for $E_{M}(t)$. This is the content of Proposition 3.1 proved in Sec. III, which states that

$$
E_{M}(t) \geq-\left(M+4\left|\Omega_{M}\right|-1\right) t \quad \text { for all } t>0
$$

Inserting this bound into (16) yields

$$
\begin{aligned}
& g(t)^{-1}\left(\frac{\sqrt{\pi}(\sqrt{2}-1)}{2} \sum_{k \geq M} l_{k}-\frac{1}{4 \sqrt{t}} \sum_{k \geq M} l_{k}^{2}+\frac{E_{M}(t)}{\sqrt{t}}\right) \\
& \geq g(t)^{-1}\left(\frac{\sqrt{\pi}(\sqrt{2}-1)}{2} \sum_{k \geq M} l_{k}-\frac{1}{4 \sqrt{t}} \sum_{k \geq M} l_{k}^{2}-\left(M+4\left|\Omega_{M}\right|-1\right) \sqrt{t}\right) .
\end{aligned}
$$

We claim that one can choose a sequence $l_{k}$ and a decreasing function $M(t)$ in such a manner that the quantity (17) tends to infinity as $t \rightarrow 0^{+}$. Indeed, setting $\tau=\sqrt{t}$ and defining $G(\tau)=g\left(\tau^{2}\right)$, the existence of such a sequence follows from Corollary 4.2 proved in Sec. IV. Note that for any $l_{k}$ and $M$ provided by Corollary 4.2 , one has necessarily $\lim _{t \rightarrow 0^{+}} M(t)=\infty$ and therefore $\left(4\left|\Omega_{M}\right|-1\right) \sqrt{t}=o(M \sqrt{t})$. Thus, for the $l_{k}$ and $M$ provided by Corollary 4.2, only the first term in (17) affects the limit. Contingent on us proving Proposition 3.1 and Corollary 4.2 , this completes the Proof of Theorem 1.1.

\section{AN ESTIMATE FOR THE ERROR TERM}

Recall that

$$
\Omega_{M}=\left\{\left(x_{1}, x_{2}\right) \in \mathbb{R}^{2}: 0<x_{1}<3,0<x_{2}<H_{M}\left(x_{1}\right)\right\}
$$

with $H_{M}$ given by (13), and $E_{M}(t)$ is defined in (15). Our goal in this section is to prove the following proposition:

Proposition 3.1. Let $\left\{l_{k}\right\}_{k \geq 1}$ be a non-negative sequence with $\sum_{k \geq 1} l_{k} \leq 1$. For every $M \geq 1$ and $t>0$,

$$
E_{M}(t) \geq-\left(M+4\left|\Omega_{M}\right|-1\right) t
$$

Remark 3.2. By comparing the bound in Proposition 3.1 with the third term in (8), the linear dependence on $M$ and $t$ in Proposition 3.1 appears to be order-sharp. Indeed, for each fixed $M$, the three-term expansion (8) states that

$$
E_{M}(t)=\frac{t}{3} \sum_{k<M}\left[\frac{\pi^{2}-(\pi / 2)^{2}}{2(\pi / 2)}+2 \frac{\pi^{2}-(5 \pi / 4)^{2}}{2(5 \pi / 4)}\right]+o(t)=\frac{\pi}{10}(M-1) t+o(t) .
$$

Let $H_{\Omega}(x, t)=e^{t \Delta_{\Omega}}(x, x)$ denote the heat kernel of the Dirichlet Laplacian on $\Omega$ evaluated on the diagonal. For our Proof of Proposition 3.1, we need a pointwise lower bound for $H_{(0, L)^{2}}(x, t)$ that has the asymptotically correct behavior close to the boundary.

Lemma 3.3. For the heat kernel of Dirichlet Laplacian on the interval $(0, L)$,

$$
H_{(0, L)}(x, t) \geq(4 \pi t)^{-1 / 2}\left(1-e^{-x^{2} / t}-e^{-(L-x)^{2} / t}\right) .
$$


Proof of Lemma 3.3. By Lemma 2.1,

$$
\begin{aligned}
H_{(0, L)}(x, t) & =\frac{1}{2 \sqrt{\pi t}} \sum_{m \in \mathbb{Z}}\left(e^{-\frac{m^{2} L^{2}}{t}}-e^{-\frac{(m L+x)^{2}}{t}}\right) \\
& =\frac{1}{2 \sqrt{\pi t}}\left[1-e^{-\frac{x^{2}}{t}}-e^{-\frac{(L-x)^{2}}{t}}+\sum_{m \geq 1}\left(2 e^{-\frac{m^{2} L^{2}}{t}}-e^{-\frac{(m L+x)^{2}}{t}}-e^{-\frac{(m L+(L-x))^{2}}{t}}\right)\right] \\
& \geq \frac{1}{2 \sqrt{\pi t}}\left[1-e^{-\frac{x^{2}}{t}}-e^{-\frac{(L-x)^{2}}{t}}\right],
\end{aligned}
$$

where in the final step, we used $e^{-\frac{m^{2} L^{2}}{t}}-e^{-\frac{(m L+x)^{2}}{t}} \geq 0$ and $e^{-\frac{m^{2} L^{2}}{t}}-e^{-\frac{(m L+(L-x))^{2}}{t}} \geq 0$ for $m \geq 1$ and $x \in(0, L)$.

Corollary 3.4. For the heat kernel of Dirichlet Laplacian on the square $(0, L)^{2}$, we have

$$
H_{(0, L)^{2}}(x, t) \geq(4 \pi t)^{-1}\left(1-e^{-d\left(x_{1}\right)^{2} / t}-e^{-d\left(x_{2}\right)^{2} / t}-4 e^{-L^{2} /(4 t)}\right),
$$

where $x=\left(x_{1}, x_{2}\right) \in(0, L)^{2}$ and $d\left(x_{j}\right)=\min \left\{x_{j}, L-x_{j}\right\}$.

Proof of Corollary 3.4. By symmetry, it suffices to consider the region where $0<x_{1}, x_{2} \leq L / 2$. By Lemma 3.3 and the product structure of the heat kernel in $(0, L)^{2}$, we have

$$
(4 \pi t) H_{(0, L)^{2}}(x, t) \geq\left(1-e^{-x_{1}^{2} / t}-e^{-\left(L-x_{1}\right)^{2} / t}\right)_{+}\left(1-e^{-x_{2}^{2} / t}-e^{-\left(L-x_{2}\right)^{2} / t}\right)_{+} .
$$

Writing $A_{+} B_{+}=\left(A+A_{-}\right)\left(B+B_{-}\right)$, we find

$$
\begin{aligned}
&\left(1-e^{-x_{1}^{2} / t}-e^{-\left(L-x_{1}\right)^{2} / t}\right)_{+}\left(1-e^{-x_{2}^{2} / t}-e^{-\left(L-x_{2}\right)^{2} / t}\right)_{+} \\
&=1-e^{-x_{1}^{2} / t}-e^{-x_{2}^{2} / t}-e^{-\left(L-x_{1}\right)^{2} / t}-e^{-\left(L-x_{2}\right)^{2} / t} \\
&+e^{-\left(x_{1}^{2}+x_{2}^{2}\right) / t}+e^{\left.-\left(x_{1}^{2}+\left(L-x_{2}\right)^{2}\right) / t\right)}+e^{-\left(\left(L-x_{1}\right)^{2}+x_{2}^{2}\right) / t} \\
&+e^{-\left(\left(L-x_{1}\right)^{2}+\left(L-x^{2}\right)^{2}\right) / t} \\
&-\left(1-e^{-x_{1}^{2} / t}-e^{-\left(L-x_{1}\right)^{2} / t}\right)\left(1-e^{-x_{2}^{2} / t}-e^{-\left(L-x_{2}\right)^{2} / t}\right)_{-} \\
&-\left(1-e^{-x_{2}^{2} / t}-e^{-\left(L-x_{2}\right)^{2} / t}\right)\left(1-e^{-x_{1}^{2} / t}-e^{-\left(L-x_{1}\right)^{2} / t}\right)_{-} \\
&+\left(1-e^{-x_{1}^{2} / t}-e^{-\left(L-x_{1}\right)^{2} / t}\right)_{-}\left(1-e^{-x_{2}^{2} / t}-e^{-\left(L-x_{2}\right)^{2} / t}\right)_{-}
\end{aligned}
$$

By discarding positive terms, using $L-x_{j} \geq L / 2$ and, since $1-e^{x_{j}^{2} / t} \geq 0$,

$$
\left(1-e^{-x_{j}^{2} / t}-e^{-\left(L-x_{j}\right)^{2} / t}\right)_{-} \leq e^{-\left(L-x_{j}\right)^{2} / t},
$$

we find

$$
\begin{aligned}
\left(1-e^{-x_{1}^{2} / t}-e^{-\left(L-x_{1}\right)^{2} / t}\right)_{+}( & \left.1-e^{-x_{2}^{2} / t}-e^{-\left(L-x_{2}\right)^{2} / t}\right)_{+} \\
\geq & 1-e^{-x_{1}^{2} / t}-e^{-x_{2}^{2} / t}-2 e^{-L^{2} /(4 t)} \\
& -\left(1-e^{-x_{2}^{2} / t}-e^{-\left(L-x_{2}\right)^{2} / t}\right)_{-}-\left(1-e^{-x_{1}^{2} / t}-e^{-\left(L-x_{1}\right)^{2} / t}\right)_{-} \\
\geq & 1-e^{-x_{1}^{2} / t}-e^{-x_{2}^{2} / t}-4 e^{-L^{2} /(4 t)},
\end{aligned}
$$

which when combined with (18) yields the claimed bound and completes the proof of the corollary.

We are now ready to prove Proposition 3.1.

Proof of Proposition 3.1. Our proof is based on the fact that if $x \in \Omega \subset \Omega^{\prime}$, then $H_{\Omega}(x, t) \leq H_{\Omega^{\prime}}(x, t)$ for any $t>0 .{ }^{18}$ We will bound $\operatorname{Tr}\left(e^{t \Delta_{\Omega_{M}}}\right)$ from below by bounding the heat kernel pointwise from below in terms of the heat kernel of appropriate squares contained in $\Omega_{M}$. 
At several points in the proof, we shall use the fact that, for $\delta, t>0$,

$$
0 \leq \frac{\sqrt{\pi t}}{2}-\int_{0}^{\delta} e^{-s^{2} / t} d s=\int_{\delta}^{\infty} e^{-s^{2} / t} d t=\frac{\sqrt{t}}{2} \int_{\delta^{2} / t}^{\infty} \frac{e^{-y}}{\sqrt{y}} d y \leq \frac{t}{2 \delta} e^{-\delta^{2} / t} .
$$

We introduce some notation for different regions in $\Omega$. Let $Q_{k}$ be the square of side-length $\sqrt{2}$ placed so that one corner matches the $k$ th tooth. By construction, each tooth is at least a distance 1 away from the vertical sides of $Q_{0}=(0,3)^{2}$. Therefore, $Q_{k} \subset \Omega_{M}$ for each $k<M$. Let $M_{k}$ be the triangular region corresponding to the $k$-th tooth, and let $L_{k}$ denote the same region but mirrored across the boundary of $Q_{0}$. The set $\overline{L_{k} \cup M_{k}}$ is a square with sidelength $l_{k} / \sqrt{2}$. Since $\frac{l_{k}}{\sqrt{2}}<\sum_{j \geq 1} \frac{l_{j}}{\sqrt{2}} \leq \frac{1}{\sqrt{2}}=\frac{\sqrt{2}}{2}$, the set $L_{k} \cup M_{k}$ is contained in one of the quarters of $Q_{k}$ obtained by cutting parallel to its sides (see Fig. 2 for an illustration of what was described above).

Writing $\operatorname{Tr}\left(e^{t \Delta_{\Omega_{M}}}\right)$ as the integral of $H_{\Omega_{M}}$, we can split the integral into pieces to be treated separately,

$$
\begin{aligned}
\operatorname{Tr}\left(e^{t \Delta_{\Omega_{M}}}\right) & =\int_{\Omega_{M}} H_{\Omega_{M}}(x, t) d x \\
& =\int_{Q_{0} \backslash \cup_{k} L_{k}} H_{\Omega_{M}}(x, t) d x+\sum_{k<M} \int_{L_{k} \cup M_{k}} H_{\Omega_{M}}(x, t) d x .
\end{aligned}
$$

For the first integral, we use $H_{\Omega_{M}}(x, t) \geq H_{Q_{0}}(x, t)$ for all $x \in Q_{0}$ and $t>0$. For the integral over $L_{k} \cup M_{k}$, we use the fact that $H_{\Omega_{M}}(x, t)$, for $x \in L_{k} \cup M_{k}$ and $t>0$, is bounded from below by $H_{Q_{k}}(x, t)$. What one finds is

$$
\operatorname{Tr}\left(e^{t \Delta_{\Omega_{M}}}\right) \geq \int_{Q_{0} \backslash \cup_{k<M} L_{k}} H_{Q_{0}}(x, t) d x+\sum_{k<M} \int_{L_{k} \cup M_{k}} H_{Q_{k}}(x, t) d x
$$

To bound the integral of $H_{Q_{0}}$, we apply Corollary 3.4, which yields, with $d(x)=\min \{x, 3-x\}$,

$$
\begin{aligned}
\int_{Q_{0} \backslash \cup_{k<M} L_{k}} H_{Q_{0}}(x, t) d x \geq & (4 \pi t)^{-1} \int_{Q_{0} \backslash \cup_{k<M} L_{k}}\left(1-e^{-d\left(x_{1}\right)^{2} / t}-e^{-d\left(x_{2}\right)^{2} / t}-4 e^{-9 /(4 t)}\right) d x \\
= & (4 \pi t)^{-1}\left|Q_{0} \backslash \cup_{k<M} L_{k}\right|\left(1-4 e^{-9 /(4 t)}\right) \\
& -(4 \pi t)^{-1} \int_{Q_{0}}\left(e^{-d\left(x_{1}\right)^{2} / t}+e^{-d\left(x_{2}\right)^{2} / t}\right) d x \\
& +(4 \pi t)^{-1} \sum_{k<M} \int_{L_{k}}\left(e^{-d\left(x_{1}\right)^{2} / t}+e^{-d\left(x_{2}\right)^{2} / t}\right) d x
\end{aligned}
$$

By symmetry and (19),

$$
\int_{Q_{0}}\left(e^{-d\left(x_{1}\right)^{2} / t}+e^{-d\left(x_{2}\right)^{2} / t}\right) d x=12 \int_{0}^{3 / 2} e^{-x_{1}^{2} / t} d x_{1} \leq 6 \sqrt{\pi t}=\frac{\sqrt{\pi t}}{2} \mathcal{H}^{1}\left(\partial Q_{0}\right)
$$

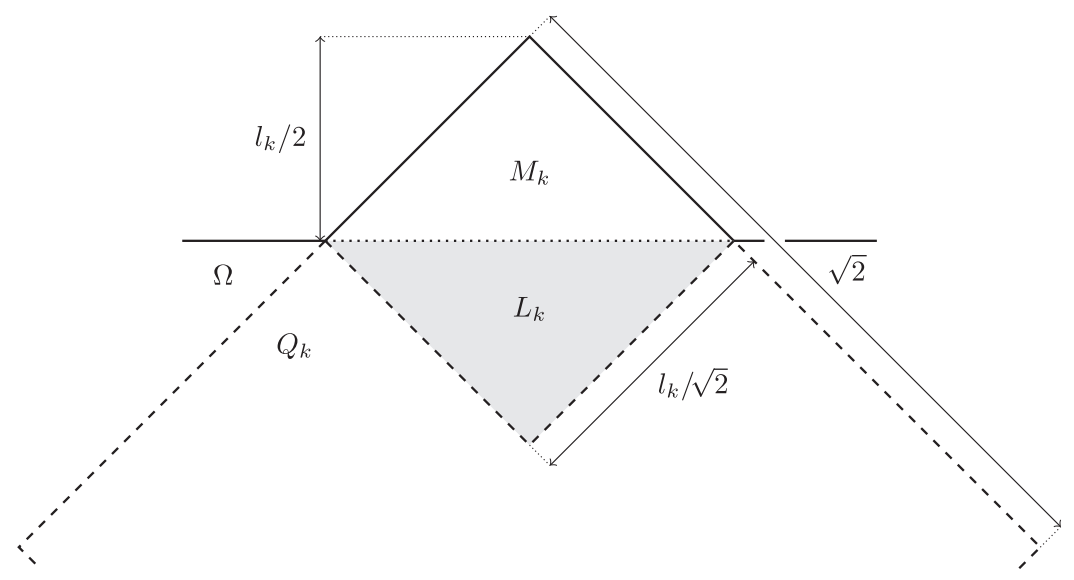

FIG. 2. Depiction of the regions $L_{k}, M_{k}$, and the corresponding square $Q_{k}$ contained in $\Omega$. 
and similarly, for any $k \geq 1$,

$$
\begin{aligned}
\int_{L_{k}}\left(e^{-d\left(x_{1}\right)^{2} / t}+e^{-d\left(x_{2}\right)^{2} / t}\right) d x & \geq \int_{L_{k}} e^{-d\left(x_{2}\right)^{2} / t} d x \\
& =\int_{0}^{l_{k} / 2}\left(l_{k}-2 s\right) e^{-s^{2} / t} d s \\
& =l_{k} \int_{0}^{l_{k} / 2} e^{-s^{2} / t} d s-t\left(1-e^{-l_{k}^{2} /(4 t)}\right) \\
& \geq \frac{\sqrt{\pi t}}{2} l_{k}-t \\
& =\frac{\sqrt{\pi t}}{2} \mathcal{H}^{1}\left(\partial L_{k} \cap \partial Q_{0}\right)-t .
\end{aligned}
$$

Thus, we have shown that

$$
\begin{aligned}
\int_{Q_{0} \backslash \cup_{k<M} L_{k}} H_{Q_{0}}(x, t) d x \geq & (4 \pi t)^{-1}\left[\left|Q_{0} \backslash \cup_{k<M} L_{k}\right|\left(1-4 e^{-9 /(4 t)}\right)\right. \\
& \left.-\frac{\sqrt{\pi t}}{2}\left(\mathcal{H}^{1}\left(\partial Q_{0}\right)-\sum_{k<M} \mathcal{H}^{1}\left(\partial L_{k} \cap \partial Q_{0}\right)\right)-(M-1) t\right] .
\end{aligned}
$$

What remains of our proof is to estimate the second integral in (20). By Corollary 3.4 and with $d\left(x_{j}\right)$ interpreted appropriately,

$$
\begin{aligned}
\int_{L_{k} \cup M_{k}} & H_{Q_{k}}(x, t) d x \\
& \geq(4 \pi t)^{-1} \int_{L_{k} \cup M_{k}}\left(1-e^{-d\left(x_{1}\right)^{2} / t}-e^{-d\left(x_{2}\right)^{2} / t}-4 e^{-1 /(2 t)}\right) d x \\
& =(4 \pi t)^{-1}\left[\left|L_{k} \cup M_{k}\right|\left(1-4 e^{-1 /(2 t)}\right)-\int_{L_{k} \cup M_{k}}\left(e^{-d\left(x_{1}\right)^{2} / t}+e^{-d\left(x_{2}\right)^{2} / t}\right) d x\right] .
\end{aligned}
$$

By symmetry, (19), and the fact that $L_{k} \cup M_{k}$ is contained in a quarter of the larger square $Q_{k}$, we have that

$$
\begin{aligned}
\int_{L_{k} \cup M_{k}}\left(e^{-d\left(x_{1}\right)^{2} / t}+e^{-d\left(x_{2}\right)^{2} / t}\right) d x & =2 \int_{L_{k} \cup M_{k}} e^{-d\left(x_{1}\right)^{2} / t} d x \\
& =\sqrt{2} l_{k} \int_{0}^{l_{k} / \sqrt{2}} e^{-s^{2} / t} d s \\
& \leq \frac{\sqrt{\pi t}}{2} \sqrt{2} l_{k} \\
& =\frac{\sqrt{\pi t}}{2} \mathcal{H}^{1}\left(\partial \Omega_{M} \cap \partial M_{k}\right) .
\end{aligned}
$$

Thus, we have shown that

$$
\int_{L_{k} \cup M_{k}} H_{Q_{k}}(x, t) d x \geq(4 \pi t)^{-1}\left[\left|L_{k} \cup M_{k}\right|\left(1-4 e^{-1 /(2 t)}\right)-\frac{\sqrt{\pi t}}{2} \mathcal{H}^{1}\left(\partial \Omega_{M} \cap \partial M_{k}\right)\right] .
$$

Combining (21) and (22), we have arrived at the bound

$$
\operatorname{Tr}\left(e^{t \Delta_{\Omega_{M}}}\right) \geq(4 \pi t)^{-1}\left[\left|\Omega_{M}\right|-\frac{\sqrt{\pi t}}{2} \mathcal{H}^{1}\left(\partial \Omega_{M}\right)-(M-1) t-4\left|\Omega_{M}\right| e^{-1 /(2 t)}\right] .
$$

Rearranging, and since $e^{-1 /(2 t)} \leq t$, for all $t \geq 0$, we have proved that

$$
E_{M}(t) \geq-\left(M+4\left|\Omega_{M}\right|-1\right) t
$$

which completes the Proof of Proposition 3.1. 


\section{AUXILIARY RESULTS}

In this section, we prove a number of technical results that will be needed in the Proof of Theorem 1.1. Specifically, we prove that one can find a function whose $L^{1}$ tail tends to zero slower than a given non-negative function $G$ while satisfying some additional properties.

Proposition 4.1. Let $G:(0,1) \rightarrow \mathbb{R}$ be a non-negative function with $\lim _{\tau \rightarrow 0^{+}} G(\tau)=0$. There exists a strictly decreasing non-negative and smooth function $h \in L^{1}((0,1))$ such that

$$
\lim _{\tau \rightarrow 0^{+}} \frac{\int_{0}^{\tau} h(x) d x}{G(\tau)}=\infty \quad \text { and } \quad \lim _{\tau \rightarrow 0^{+}} \frac{\tau h(\tau)+\frac{1}{\tau} \int_{0}^{\tau} x h(x) d x}{\int_{0}^{\tau} h(x) d x}=0 .
$$

As a consequence of Proposition 4.1, we can prove the following result in the setting of sequences, which provides the final ingredient to complete the Proof of Theorem 1.1:

Corollary 4.2. Let $G:(0,1) \rightarrow \mathbb{R}$ be a non-negative function with $\lim _{\tau \rightarrow 0^{+}} G(\tau)=0$ and let $A>0$. There exists a positive non-increasing sequence $\left\{l_{k}\right\}_{k \geq 1}$ with $\sum_{k \geq 1} l_{k}=A$ and a decreasing function $M:(0,1) \rightarrow \mathbb{N}$ such that

$$
\lim _{\tau \rightarrow 0^{+}} \frac{\sum_{k \geq M(\tau)} l_{k}}{G(\tau)}=\infty \quad \text { and } \quad \lim _{\tau \rightarrow 0^{+}} \frac{\tau M(\tau)+\frac{1}{\tau} \sum_{k \geq M(\tau)} l_{k}^{2}}{\sum_{k \geq M(\tau)} l_{k}}=0 .
$$

We first prove Proposition 4.1 and then show how to deduce Corollary 4.2 from it. To simplify the Proof of Proposition 4.1 , it will be convenient to first show that we may assume that $G$ is fairly well-behaved.

Lemma 4.3. Let $G:(0,1) \rightarrow \mathbb{R}$ be a bounded non-negative function with $\lim _{\tau \rightarrow 0^{+}} G(\tau)=0$. There exists a function $\widehat{G} \in C^{\infty}((0,1))$ such that

(a) $\lim _{\tau \rightarrow 0^{+}} \widehat{G}(\tau)=0$,

(b) $\widehat{G}(\tau)>G(\tau)$ for all $\tau \in(0,1)$,

(c) $\widehat{G}^{\prime}(\tau)>0$ for all $\tau \in(0,1)$, and

(d) $\widehat{G}^{\prime \prime}(\tau)<0$ for all $\tau \in(0,1)$.

Proof of Lemma 4.3. Fix $G$ as in the lemma. Define, for $\tau>0$,

$$
\bar{G}(\tau)=\inf \{a \tau+b: a \geq 0, b \geq 0, G(s) \leq a s+b \text { for all } s \in(0,1)\} .
$$

It is clear from the construction that $\bar{G}$ is non-decreasing, concave, and satisfies $G(\tau) \leq \bar{G}(\tau)$ for all $\tau \in(0,1)$. This $\lim _{\tau \rightarrow 0^{+}} \bar{G}(\tau)=0$ follows if we can prove that for every $b>0$, there exists $a \geq 0$ such that $G(s) \leq a s+b$ for all $s \in[0,1]$. Since $G(s)-b$ is negative for $s$ small enough, the choice $a=\sup _{0<s<1} \frac{(G(s)-b)_{+}}{s}$ works.

Fix $\varphi \in C_{0}^{\infty}((1,2))^{s}$ non-negative with $\int_{\mathbb{R}} \varphi(x) d x=1$. Let

$$
\tilde{G}(\tau)=\int_{\mathbb{R}} \varphi(x) \bar{G}(\tau x) d x=\frac{1}{\tau} \int_{\mathbb{R}} \varphi(y / \tau) \bar{G}(y) d y .
$$

Since $\varphi \in C_{0}^{\infty}((1,2))$, it holds that $\tilde{G} \in C^{\infty}((0,1))$. Since $\varphi \geq 0$, supp $\varphi \subseteq[1,2]$, and $\bar{G}$ is non-decreasing,

$$
\tilde{G}(\tau)=\int_{\mathbb{R}} \varphi(x) \bar{G}(\tau x) d x \geq \bar{G}(\tau) \geq G(\tau)
$$

and similarly,

$$
\lim _{\tau \rightarrow 0^{+}} \tilde{G}(\tau)=\lim _{\tau \rightarrow 0^{+}} \int_{\mathbb{R}} \varphi(x) \bar{G}(\tau x) d x \leq \lim _{\tau \rightarrow 0^{+}} \bar{G}(2 \tau)=0 .
$$

Furthermore, $\tilde{G}$ is increasing and concave, both of which are consequences of the corresponding properties for $\bar{G}$. Indeed, for $0<\tau_{1}<\tau_{2} \leq 1$,

$$
\tilde{G}\left(\tau_{2}\right)-\tilde{G}\left(\tau_{1}\right)=\int_{\mathbb{R}} \varphi(x)\left(\bar{G}\left(\tau_{2} x\right)-\bar{G}\left(\tau_{1} x\right)\right) d x \geq 0,
$$

and for any $\alpha \in(0,1)$,

$$
\begin{aligned}
\tilde{G}\left((1-\alpha) \tau_{1}+\alpha \tau_{2}\right) & =\int_{\mathbb{R}} \varphi(x)\left(\bar{G}\left((1-\alpha) \tau_{1} x+\alpha \tau_{2} x\right)\right) d x \\
& \geq \int_{\mathbb{R}} \varphi(x)\left((1-\alpha) \bar{G}\left(\tau_{1} x\right)+\alpha \bar{G}\left(\tau_{2} x\right)\right) d x \\
& =(1-\alpha) \tilde{G}\left(\tau_{1}\right)+\alpha \tilde{G}\left(\tau_{2}\right) .
\end{aligned}
$$


From (23)-(26), it follows that $\hat{G}(\tau)=\tilde{G}(\tau)+\sqrt{\tau}$ satisfies the properties claimed in the lemma.

Proof of Proposition 4.1. Clearly we can assume that $G$ is bounded. By Lemma 4.3, we can without loss of generality assume that $G$ is smooth, strictly increasing, and concave.

Set $h(\tau)=G^{\prime}(\tau) U(G(\tau))$ for some non-negative, integrable, and strictly decreasing $U \in C^{\infty}\left(\left(0,\|G\|_{\infty}\right)\right)$ to be specified. By the assumptions on $G, U$, we have $h \in C^{\infty}$ and, for $0<\tau_{1}<\tau_{2} \leq 1$,

$$
h\left(\tau_{1}\right)-h\left(\tau_{2}\right)=G^{\prime}\left(\tau_{1}\right) U\left(G\left(\tau_{1}\right)\right)-G^{\prime}\left(\tau_{2}\right) U\left(G\left(\tau_{2}\right)\right)>\left(G^{\prime}\left(\tau_{1}\right)-G^{\prime}\left(\tau_{2}\right)\right) U\left(G\left(\tau_{1}\right)\right) \geq 0,
$$

and thus, $h$ is strictly decreasing. Moreover, $h$ is integrable since

$$
\int_{0}^{1} h(\tau) d \tau=\int_{0}^{1} G^{\prime}(\tau) U(G(\tau)) d \tau=\int_{0}^{G(1)} U(y) d y<\infty .
$$

By the same computation, we have that

$$
\int_{0}^{\tau} h(x) d x=\int_{0}^{G(\tau)} U(y) d y
$$

and as such, we see that in order to satisfy the first claim of the proposition, we simply need to choose $U$ so that $\int_{0}^{s} U(y) d y \gg s$.

Since $G$ is increasing and concave, we can bound

$$
G(\tau)=\int_{0}^{\tau} G^{\prime}(x) d x \geq \tau G^{\prime}(\tau)
$$

Using this, we wish to estimate the terms in the numerator of the second limit.

Since $U \geq 0$, the inequality (28) implies that

$$
\tau h(\tau)=\tau G^{\prime}(\tau) U(G(\tau)) \leq G(\tau) U(G(\tau))
$$

For the second term, an integration by parts yields

$$
\begin{aligned}
\frac{1}{\tau} \int_{0}^{\tau} x h(x) d x & =\frac{1}{\tau} \int_{0}^{\tau} x G^{\prime}(x) U(G(x)) d x \\
& =-\frac{1}{\tau} \int_{0}^{\tau}\left[G(x) U(G(x))+x G(x) G^{\prime}(x) U^{\prime}(G(x))\right] d x+G(\tau) U(G(\tau)),
\end{aligned}
$$

where we used $\lim _{x \rightarrow 0^{+}} x G(x) U(G(x))=0$ by integrability and monotonicity of $U$. Using again (28) to bound the first term in the brackets, one finds

$$
\begin{aligned}
\frac{1}{\tau} \int_{0}^{\tau} x h(x) d x & \leq-\frac{1}{\tau} \int_{0}^{\tau}\left[x G^{\prime}(x) U(G(x))+x G(x) G^{\prime}(x) U^{\prime}(G(x))\right] d x+G(t) U(G(\tau)) \\
& =-\frac{1}{\tau} \int_{0}^{\tau} x G^{\prime}(x)\left[U(G(x))+G(x) U^{\prime}(G(x))\right] d x+G(\tau) U(G(\tau)) .
\end{aligned}
$$

Let $U(y)=y^{-1}\left(\log \frac{e^{2}\|G\|_{\infty}}{y}\right)^{-2}$. Note that, for $y \in\left(0,\|G\|_{\infty}\right)$,

$$
U^{\prime}(y)=-\frac{U(y)}{y}\left(1-2\left(\log \frac{e^{2}\|G\|_{\infty}}{y}\right)^{-1}\right)<0 \quad \text { for all } 0<y<\|G\|_{\infty} .
$$

Moreover,

$$
\begin{aligned}
& \int_{0}^{G(\tau)} U(y) d y=\left(\log \frac{e^{2}\|G\|_{\infty}}{G(\tau)}\right)^{-1}, \\
& G(\tau) U(G(\tau))=\left(\log \frac{e^{2}\|G\|_{\infty}}{G(\tau)}\right)^{-2}, \text { and } \\
& U(y)+y U^{\prime}(y)=2 y^{-1}\left(\log \frac{e^{2}\|G\|_{\infty}}{y}\right)^{-3}>0 \text { for all } 0<y<\|G\|_{\infty} .
\end{aligned}
$$

Thus, by (30) and (29),

$$
\tau h(\tau)+\frac{1}{\tau} \int_{0}^{\tau} x h(x) d x \leq \tau h(\tau)+G(\tau) U(G(\tau)) \leq 2 G(\tau) U(G(\tau))
$$


Combining (27) and (29) with the equations in (31), we finally obtain that

$$
\lim _{\tau \rightarrow 0^{+}} \frac{\int_{0}^{\tau} h(x) d x}{G(\tau)}=\lim _{\tau \rightarrow 0^{+}} G(\tau)^{-1}\left(\log \frac{e^{2}\|G\|_{\infty}}{G(\tau)}\right)^{-1}=\infty,
$$

and similarly, by (32),

$$
0 \leq \lim _{\tau \rightarrow 0^{+}} \frac{\tau h(\tau)+\frac{1}{\tau} \int_{0}^{\tau} x h(x) d x}{\int_{0}^{\tau} h(x) d x} \leq \lim _{\tau \rightarrow 0^{+}} 2\left(\log \frac{e^{2}\|G\|_{\infty}}{G(\tau)}\right)^{-1}=0,
$$

which concludes the proof of the proposition.

Proof of Corollary 4.2. Without loss of generality, we can assume that $G(\tau) \geq \tau$. By Proposition 4.1, there exists a non-negative strictly decreasing smooth function $h \in L^{1}((0,1))$ such that

$$
\lim _{\tau \rightarrow 0^{+}} \frac{\int_{0}^{\tau} h(x) d x}{G(\tau)}=\infty \quad \text { and } \quad \lim _{\tau \rightarrow 0^{+}} \frac{\tau h(\tau)+\frac{1}{\tau} \int_{0}^{\tau} x h(x) d x}{\int_{0}^{\tau} h(x) d x}=0 .
$$

The assumption that $G(\tau) \geq \tau$ implies that $\lim _{\tau \rightarrow 0^{+}} h(\tau)=\infty$.

Since $h$ is strictly decreasing, we can consider its inverse, $h^{-1}:(h(1), \infty) \rightarrow \mathbb{R}$, which is again strictly decreasing. Define $f:(0, \infty) \rightarrow \mathbb{R}$ by $f(y)=h^{-1}(y) \mathbb{1}_{y>h(1)}+\mathbb{1}_{y \leq h(1)}$. Then,

$$
\int_{0}^{\infty} f(y) d y=h(1)+\int_{h(1)}^{\infty} h^{-1}(y) d y=h(1)-\int_{0}^{1} x h^{\prime}(x) d x=\int_{0}^{1} h(x) d x
$$

where we used the fact that $h$ is integrable and monotonic to conclude that $\lim _{\tau \rightarrow 0^{+}} \tau h(\tau)=0$. Since the equation $f(y)=\tau$ has a unique solution for each $\tau \in(0,1)$, the inverse of $f^{-1}:(0,1) \rightarrow \mathbb{R}$ is well-defined, and by construction, $f^{-1}(\tau)=h(\tau)$.

Since $f$ is decreasing and integrable, $\sum_{k \geq 1} f(k)<\infty$. Set $l_{k}=c_{0} f(k)$ with $c_{0}$ chosen so that $\sum_{k \geq 1} l_{k}=A$. Define $M(\tau)$ to be the smallest integer such that $M(\tau) \geq f^{-1}(\tau)=h(\tau)$. To complete the proof of the corollary, we need to relate the quantities in the statement to the corresponding quantities in Proposition 4.1.

Since $f$ is monotonically decreasing, we can estimate

$$
\begin{aligned}
& \sum_{k \geq M(\tau)} f(k) \geq \int_{M(\tau)}^{\infty} f(y) d y=\int_{h(\tau)}^{\infty} f(y) d y-\int_{h(\tau)}^{M(\tau)} f(y) d y \geq \int_{h(\tau)}^{\infty} f(y) d y-\tau, \\
& \sum_{k \geq M(\tau)} f^{2}(y) \leq \int_{M(\tau)}^{\infty} f^{2}(y) d y+f^{2}(M(\tau)) \leq \int_{h(\tau)}^{\infty} f^{2}(y) d y+\tau^{2} .
\end{aligned}
$$

By the change of variables $x=f(y)$, i.e., $y=h(x)$, and an integration by parts,

$$
\int_{h(\tau)}^{\infty} f(y) d y=-\int_{0}^{\tau} x h^{\prime}(x) d x=\int_{0}^{\tau} h(x) d x-\tau h(\tau),
$$

and similarly,

$$
\int_{h(\tau)}^{\infty} f^{2}(y) d y=-\int_{0}^{\tau} x^{2} h^{\prime}(x) d x=2 \int_{0}^{\tau} x h(x) d x-\tau^{2} h(\tau) .
$$

Combining (33)-(36) with the properties of $h$ in Proposition 4.1 and $G(\tau) \geq \tau$, we find

$$
\lim _{\tau \rightarrow 0^{+}} \frac{\sum_{k \geq M(\tau)} l_{k}}{G(\tau)} \geq c_{0} \lim _{\tau \rightarrow 0^{+}} \frac{\int_{0}^{\tau} h(x) d x-\tau(h(\tau)+1)}{G(\tau)}=\infty,
$$

and since $M(\tau) \leq h(\tau)+1$,

$$
0 \leq \lim _{\tau \rightarrow 0^{+}} \frac{\tau M(\tau)+\frac{1}{\tau} \sum_{k \geq M(\tau)} l_{k}^{2}}{\sum_{k \geq M(\tau)} l_{k}} \leq \lim _{\tau \rightarrow 0^{+}} \frac{c_{0}^{-1} \tau(h(\tau)+1)+\frac{c_{0}}{\tau}\left(2 \int_{0}^{\tau} x h(x) d x+\tau^{2}\right)}{\int_{0}^{\tau} h(x) d x-\tau(h(\tau)+1)}=0 .
$$

This completes the Proof of Corollary 4.2. 


\section{PROOF OF THEOREM 1.2}

In the final section of this paper, we provide a proof that Theorem 1.1 implies Theorem 1.2.

Proof of Theorem 1.2. Fix $R$ as in the theorem. Without loss of generality, we may assume that $R$ is bounded.

Let

$$
g_{0}(t)=\int_{0}^{\infty} \mu^{(d-1) / 2} R(\mu / t) e^{-\mu / 2} d \mu
$$

and $\tilde{g}_{0}(t)=\max \left\{g_{0}(t), t^{(d+1) / 2}\right\}$. By dominated convergence, $\lim _{t \rightarrow 0^{+}} \tilde{g}_{0}(t)=0$. By Theorem 1.1, there exists an open, bounded, and connected Lipschitz regular set $\Omega_{0} \subset \mathbb{R}^{d}$ such that

$$
\limsup _{t \rightarrow 0^{+}} \frac{(4 \pi t)^{d / 2} \operatorname{Tr}\left(e^{t \Delta_{\Omega_{0}}}\right)-\left|\Omega_{0}\right|+\frac{\sqrt{\pi t}}{2} \mathcal{H}^{d-1}\left(\partial \Omega_{0}\right)}{\sqrt{t} \tilde{g}_{0}(t)}=\infty .
$$

Assume that the conclusion of Theorem 1.2 is wrong. Then, there exist $C<\infty, \gamma \geq 0$, and $0 \leq \lambda_{0}<\infty$ such that for all $\lambda \geq \lambda_{0}$,

$$
\operatorname{Tr}\left(-\Delta_{\Omega_{0}}-\lambda\right)_{-}^{\gamma}-L_{\gamma, d}\left|\Omega_{0}\right| \lambda^{\gamma+d / 2}+\frac{L_{\gamma, d-1}}{4} \mathcal{H}^{d-1}\left(\partial \Omega_{0}\right) \lambda^{\gamma+(d-1) / 2} \leq C \lambda^{\gamma+(d-1) / 2} R(\lambda) .
$$

By (6), we conclude that

$$
\begin{aligned}
& \operatorname{Tr}\left(e^{t \Delta_{\Omega_{0}}}\right)-(4 \pi t)^{-d / 2}\left|\Omega_{0}\right|+\frac{\sqrt{\pi t}}{2}(4 \pi t)^{-d / 2} \mathcal{H}^{d-1}\left(\partial \Omega_{0}\right) \\
& =\frac{t^{1+\gamma}}{\Gamma(1+\gamma)} \int_{0}^{\infty}\left[\operatorname{Tr}\left(-\Delta_{\Omega_{0}}-\lambda\right)_{-}^{\gamma}-L_{\gamma, d}\left|\Omega_{0}\right| \lambda^{\gamma+d / 2}+\frac{L_{\gamma, d-1}}{4} \mathcal{H}^{d-1}\left(\partial \Omega_{0}\right) \lambda^{\gamma+(d-1) / 2}\right] e^{-t \lambda} d \lambda \\
& \leq \frac{t^{1+\gamma}}{\Gamma(1+\gamma)}\left[C \int_{\lambda_{0}}^{\infty} \lambda^{\gamma+(d-1) / 2} R(\lambda) e^{-t \lambda} d \lambda+c(t)\right],
\end{aligned}
$$

where we write

$$
c(t)=\int_{0}^{\lambda_{0}}\left[\operatorname{Tr}\left(-\Delta_{\Omega_{0}}-\lambda\right)_{-}^{\gamma}-L_{\gamma, d}\left|\Omega_{0}\right| \lambda^{\gamma+d / 2}+\frac{L_{\gamma, d-1}}{4} \mathcal{H}^{d-1}\left(\partial \Omega_{0}\right) \lambda^{\gamma+(d-1) / 2}\right] e^{-t \lambda} d \lambda .
$$

Using the fact that $\mu^{\gamma} e^{-\mu / 2} \leq\left(\frac{2 \gamma}{e}\right)^{\gamma}$ for all $\mu \geq 0$,

$$
\begin{aligned}
t^{1+\gamma} \int_{\lambda_{0}}^{\infty} \lambda^{\gamma+(d-1) / 2} R(\lambda) e^{-t \lambda} d \lambda & =t^{-(d-1) / 2} \int_{\lambda_{0} t}^{\infty} \mu^{\gamma+(d-1) / 2} R(\mu / t) e^{-\mu} d \mu \\
& \leq\left(\frac{2 \gamma}{e}\right)^{\gamma} t^{-(d-1) / 2} \int_{\lambda_{0} t}^{\infty} \mu^{(d-1) / 2} R(\mu / t) e^{-\mu / 2} d \mu \\
& \leq\left(\frac{2 \gamma}{e}\right)^{\gamma} t^{-(d-1) / 2} g_{0}(t) \\
& \leq\left(\frac{2 \gamma}{e}\right)^{\gamma} t^{-(d-1) / 2} \tilde{g}_{0}(t)
\end{aligned}
$$

Moreover, we bound $c(t)$ by discarding the negative volume term and use monotonicity to find

$$
\begin{aligned}
c(t) & \leq\left(\operatorname{Tr}\left(-\Delta_{\Omega_{0}}-\lambda_{0}\right)_{-}^{\gamma}+\frac{L_{\gamma, d-1}}{4} \mathcal{H}^{d-1}\left(\partial \Omega_{0}\right) \lambda_{0}^{\gamma+(d-1) / 2}\right) \int_{0}^{\lambda_{0}} e^{-t \lambda} d \lambda \\
& \leq\left(\operatorname{Tr}\left(-\Delta_{\Omega_{0}}-\lambda_{0}\right)_{-}^{\gamma}+\frac{L_{\gamma, d-1}}{4} \mathcal{H}^{d-1}\left(\partial \Omega_{0}\right) \lambda_{0}^{\gamma+(d-1) / 2}\right) \lambda_{0} .
\end{aligned}
$$

By construction, $\tilde{g}_{0}(t) \geq t^{(d+1) / 2}$, and therefore,

$$
\limsup _{t \rightarrow 0^{+}} t^{\gamma+(d+1) / 2} \tilde{g}_{0}(t)^{-1} c(t)<\infty
$$

Thus, we have shown that

$$
\limsup _{t \rightarrow 0^{+}} \frac{(4 \pi t)^{d / 2} \operatorname{Tr}\left(e^{t \Omega_{\Omega_{0}}}\right)-\left|\Omega_{0}\right|+\frac{\sqrt{\pi t}}{2} \mathcal{H}^{d-1}\left(\partial \Omega_{0}\right)}{\sqrt{t} \tilde{g}_{0}(t)}<\infty,
$$

contradicting the choice of $\Omega_{0}$. This completes the Proof of Theorem 1.2. 


\section{ACKNOWLEDGMENTS}

We would like to dedicate this paper to the memory of Jean Bourgain. The U.S. National Science Foundation (Grant No. DMS-1363432) (R.L.F.) and Knut and Alice Wallenberg Foundation (Grant No. KAW 2018.0281) (S.L.) are acknowledged. The authors also wish to thank Institut Mittag-Leffler, where part of this work was carried out.

Data sharing is not applicable to this article as no new data were created or analyzed in this study.

\section{REFERENCES}

${ }^{1} \mathrm{H}$. Weyl, "Das asymptotische verteilungsgesetz der eigenwerte linearer partieller differentialgleichungen (mit einer Anwendung auf die Theorie der Hohlraumstrahlung)," Math. Ann. 71(4), 441-479 (1912).

${ }^{2}$ G. V. Rozenblum, "On the eigenvalues of the first boundary value problem in unbounded domains," Math. USSR-Sb. 18, 235-248 (1973).

${ }^{3} \mathrm{H}$. Weyl, "Über die randwertaufgabe der strahlungstheorie und asymptotische spektralgesetze," J. Reine Angew. Math. 1913(143), 177-202.

${ }^{4} \mathrm{~V}$. Ivrii, “The second term of the spectral asymptotics for a Laplace-Beltrami operator on manifolds with boundary," Funkt. Anal. i Prilozhen. 14(2), 25-34 (1980).

${ }^{5}$ R. M. Brown, "The trace of the heat kernel in Lipschitz domains," Trans. Am. Math. Soc. 339(2), 889-900 (1993).

${ }^{6}$ R. L. Frank and S. Larson, "Two-term spectral asymptotics for the Dirichlet Laplacian in a Lipschitz domain," J. Reine Angew. Math. (published online).

${ }^{7}$ V. F. Lazutkin and D. Ya. Terman, "Estimation of the remainder in the Weyl formula," Funct. Anal. Appl. 15(4), 299-300 (1982).

${ }^{8}$ M. van den Berg and S. Srisatkunarajah, "Heat equation for a region in $\mathbf{R}^{2}$ with a polygonal boundary," J. London Math. Soc. s2-37(1), 119-127 (1988).

${ }^{9}$ B. V. Fedosov, "Asymptotic formulae for the eigenvalues of the Laplace operator in the case of a polygonal domain," Dokl. Akad. Nauk SSSR 151(4), 786-789 (1963).

${ }^{10}$ M. Kac, "Can one hear the shape of a drum?," Am. Math. Monthly 73(4), 1-23 (1966), part II.

${ }^{11}$ R. Mazzeo and J. Rowlett, "A heat trace anomaly on polygons," Math. Proc. Cambridge Philos. Soc. 159(2), 303-319 (2015).

${ }^{12}$ H. P. McKean, Jr. and I. M. Singer, "Curvature and the eigenvalues of the Laplacian," J. Differ. Geom. 1(1), 43-69 (1967).

${ }^{13}$ B. V. Fedosov, "Asymptotic formulae for the eigenvalues of the Laplace operator for a polyhedron," Dokl. Akad. Nauk SSSR 157(3), 536-538 (1964).

${ }^{14}$ M. V. Berry, "Distribution of modes in fractal resonators," in Structural Stability in Physics, edited by W. Güttinger and H. Eikemeier (Springer-Verlag, Berlin, 1979), pp. 51-53.

${ }^{15}$ M. V. Berry, "Some geometric aspects of wave motion: Wavefront dislocations, diffraction catastrophes, diffractals," in Geometry of the Laplace Operator (Proc. Sympos. Pure Math., Univ. Hawaii, Honolulu, HI, 1979), Proc. Sympos. Pure Math., XXXVI (Amer. Math. Soc., Providence, RI, 1980), pp. $241-252$.

${ }^{16}$ J. Brossard and R. Carmona, "Can one hear the dimension of a fractal?," Commun. Math. Phys. 104(1), 103-122 (1986).

${ }^{17}$ M. L. Lapidus, "Fractal drum, inverse spectral problems for elliptic operators and a partial resolution of the Weyl-Berry conjecture," Trans. Am. Math. Soc. 325(2), 465-529 (1991).

${ }^{18}$ E. B. Davies, Heat Kernels and Spectral Theory, Cambridge Tracts in Mathematics Vol. 92 (Cambridge University Press, Cambridge, 1989). 